\title{
Préparer à communiquer par écrit dans l'entreprise : courrier traditionnel vs courrier électronique
}

\section{Elzbieta Gajewska}

\section{OpenEdition}

\section{Journals}

Édition électronique

URL : http://journals.openedition.org/pratiques/3231

DOI : 10.4000/pratiques.3231

ISSN : 2425-2042

Éditeur

Centre de recherche sur les médiations (CREM)

Référence électronique

Elzbieta Gajewska, «Préparer à communiquer par écrit dans l'entreprise : courrier traditionnel vs courrier électronique », Pratiques [En ligne], 171-172 | 2016, mis en ligne le 07 février 2017, consulté le 30 avril 2019. URL : http://journals.openedition.org/pratiques/3231 ; DOI : 10.4000/pratiques.3231

Ce document a été généré automatiquement le 30 avril 2019.

(c) Tous droits réservés 


\title{
Préparer à communiquer par écrit dans l'entreprise : courrier traditionnel vs courrier électronique
}

\author{
Elzbieta Gajewska
}

1 À partir des années 1990, nous assistons à un bouleversement des pratiques d'écriture lié à l'avènement des médias électroniques. Cette révolution se manifeste aussi au niveau des outils pour la formation en communication d'entreprise.

2 Communiquer efficacement par écrit en contexte et pour des besoins professionnels est un savoir-faire qui s'apprend. Pour cette raison, par le passé, dans les entreprises, la rédaction du courrier était allouée au personnel ayant une formation spécialisée (secrétaires ou adjoints). Avec l'avènement des logiciels de messagerie, savoir rédiger devient indispensable à un nombre de plus en plus large de professionnels. Or, si les rédacteurs du courrier traditionnel étaient assistés dans leur tâche par maintes guides ou recueils de "modèles de lettres ", les outils censés faciliter la rédaction du courrier électronique sont de loin moins nombreux et exigent de la part de leurs utilisateurs nettement plus d'application et de compétence.

3 Quand les missives commerciales étaient encore confiées aux bons soins de la poste, le noyau dur des guides pour leur rédaction était constitué par des «modèles de lettres » (ou « lettres types »), accompagnés (ou pas) des suggestions comment adapter le texte en question à une situation donnée. Les conseils d'ordre général, placés en introduction, étaient fort limités et portaient avant tout sur le style.

4 La popularisation des logiciels de traitement de texte a eu pour conséquence une montée en vague des cédéroms avec des documents prêts à imprimer. Paradoxalement, la généralisation des messageries électroniques a contribué au déclin de ces outils. Les manuels pour la rédaction du courrier professionnel électronique non seulement n'égalent pas en nombre leurs ancêtres (fait qui aurait pu s'expliquer par l'abandon des manuels en papier au profit des e-books), mais de plus semblent avoir des proportions 
inversées par rapport aux guides qui initiaient au courrier traditionnel: les conseils d'ordre général y sont accompagnés des exemples de courriels, qui pourtant ne sont pas présentés comme des «modèles » de communication dans tel ou tel contexte situationnel, mais comme des illustrations exemplifiant telle ou telle réflexion ${ }^{1}$.

La même tendance se dessine si nous orientons notre recherche vers les ressources de l'Internet. S'il existe des sites qui proposent des centaines de lettres commerciales ou administratives à télécharger, les pages web dédiées au courriel misent davantage sur des conseils rédactionnels que sur des "modèles" à imiter. Cet abandon d'un format pourtant bien pratique et utilisé pendant des décennies semble témoigner d'un changement plus profond. Par la suite, nous allons examiner ce phénomène par le biais du genre et chercher son explication dans les changements génériques qu'il rend manifestes, tout en essayant d'en tirer des indications didactiques.

6 Afin de restreindre notre champ d'investigation, nous allons nous recentrer sur le courrier et le courriel commercial, qui constituent une partie essentielle de la communication externe de l'entreprise. En premier lieu, nous allons examiner l'impact du nouveau moyen de transmettre le message sur sa forme et le potentiel générique qui en résulte (1). Cette nouvelle donne qu'est la transmission électronique peut en effet non seulement affecter des rôles nouveaux à des éléments repris au courrier traditionnel (1.1), mais également exposer le document écrit, jusqu'à présent immuable, à des «remaniements» d'ordre divers qui n'étaient pas possibles auparavant, lequel phénomène ne facilite certes pas la stabilisation en genres (1.2). Par la suite, nous allons élargir notre champ d'examen - du texte à toute la situation de communication en entreprise dont il résulte - afin d'établir comment les outils électroniques reconfigurent les pratiques communicatives (2). Nous allons examiner la manière dont les missives professionnelles sont imbriquées dans l'action répétitive qui les formate en genres (2.1), avant de réfléchir sur les changements advenus et leurs conséquences pour les pratiques communicatives, notamment sur les potentialités de stabilisation des nouvelles modes de communiquer en genres (2.2). Dans le cadre de ces réflexions, nous allons également envisager si des catégories plus larges que le genre (3) pourraient éventuellement servir de repère lors de l'enseignement et l'apprentissage de l'art du « courriel professionnel » (3.2.), même si de telles catégories étaient d'une utilité restreinte par rapport au courrier traditionnel (3.1).

\section{Courrier professionnel vs courriel professionnel : la médiation de la technologie et son potentiel générique}

7 Les particularités de la communication médiatisée par ordinateur (CEMO) et de ses genres ont fait couler beaucoup d'encre et il serait impossible de les présenter de manière exhaustive dans le cadre du présent article, même en nous limitant aux commentaires formulés précisément par rapport aux messages électroniques. Nous allons donc par la suite juste signaler quelques incidences de la technologie sur des messages rédigés en contexte professionnel, à partir desquelles nous voulons mettre en relief l'impact de cette nouvelle donne sur les propriétés génériques du courriel « d'affaires ».

8 Les traits particuliers de la communication écrite d'entreprise sont, d'après Girault (1996 : 4), son efficacité et sa valeur de preuve légale. La permanence de l'information évite la déformation du message (qui peut être relu et utilisé plusieurs fois) et constitue un 
moyen de preuve en cas de contestation. Ainsi donc, le texte doit être aisé à comprendre et lever la moindre ambiguité, postulats qui ont influencé la présentation et le contenu du courrier d'entreprise. Parmi les solutions pratiques à ces exigences, nous pouvons citer le «normolettre» et les dispositifs de mise en texte des messageries qui organisent le message en fonction d'un schéma préformé. Bien que la structure pré-formatée du courriel reprend certains éléments des lettres papier, un mode de transmission différent a entraîné la reconfiguration des fonctions traditionnellement assignées à ces éléments dans la communication entre les sociétés. De plus, par rapport au support ancien, le nouveau médium offre des potentialités jusqu'à présent inconnues.

\subsection{Des rôles nouveaux pour des éléments anciens}

9 Un canal de communication différent contribue à la constitution de pratiques nouvelles, qui peuvent par la suite se stabiliser en genres. Comme la réception de la lettre a lieu un certain temps après sa rédaction et se fait en l'absence de son émetteur, la tradition épistolaire a créé un dispositif complexe qui, à côté du texte principal, fournit des informations sur le contexte de son énonciation. Par rapport à la dichotomie énonciation directe vs énonciation indirecte, le courriel se trouve dans une situation intermédiaire, «car s'il y a énonciation directe et réception présupposée imminente, la lecture, l'appropriation par l'allocutaire n'est pas instantanée » (Cusin-Berche 1999: 33). L'auteur du texte n'apparaît pour le récepteur qu'à travers des procédés d'identification écrite. Ainsi, tout comme dans la communication épistolaire traditionnelle, un dispositif assurant la transmission des informations contextuelles fait partie inhérente de la messagerie électronique.

10 Mourlhon-Dallies \& Colin (1999) appliquent ici la notion de « paratexte » qui désigne tout ce qui entoure et prolonge un texte sans être le texte proprement dit. Le paratexte d'une lettre, traditionnelle ou électronique, permet de situer le contexte de l'énonciation: le destinateur et le destinataire, le temps et le lieu de rédaction. Le paratexte est aussi un moyen qui aide le lecteur (Guérin, 2008) : par une série de signaux, il met en place une stratégie destinée à organiser la réception du texte. Les messages publicitaires (dont lettre de vente) à part ${ }^{2}$, le courrier d'entreprise et des affaires a des objectifs fonctionnels, où la mise en page est au service d'une interprétation facile et univoque. Toutefois, vu que le format papier et celui électronique diffèrent considérablement, ces différences vont se refléter dans le normolettre et son équivalent numérique.

11 Contrastée au courrier classique, la lettre numérique apparaît davantage comme un outil de communication efficace qu'en tant que preuve légale. Ainsi, certains éléments du normolettre électronique ont gagné en importance et tendent à devenir des composantes rhétoriques stratégiques. Un exemple probant en est la signature. La signature manuscrite et dactylographiée du courrier traditionnel est aujourd'hui remplacée dans les missives électroniques par la signature tapuscrite (selon l'appellation de CuisinBerche, 1999) et par la signature insérée de manière automatique, à ne pas confondre avec la signature électronique. Toutefois, entre les anciennes et les nouvelles formes il n'y a pas de corrélation directe. Contrastée à celle du courrier d'entreprise traditionnel, la signature utilisée dans le courriel s'avère tout d'abord une signature qui se décline au pluriel et prend des formes variées. Dans le courriel d'entreprise, la signature tapuscrite peut prendre des formes variées en fonction du contexte (nom+prénom, prénom, nom +prénom+fonction), apportant par ceci au document une touche personnalisée. Pour cette 
raison, Mourlhon-Dallies \& Colin (1999) envisagent la signature comme une partie du texte du message, et non de son paratexte liminaire.

Dans le courrier diffusé sur papier, la signature apportée « de sa propre main » donnait à l'écrit la valeur de preuve légale, les informations dactylographiées apportant des information complémentaires (p.ex. sur la fonction du signant). Dans le courriel, si elle perd de sa valeur d'authentification du document (cette fonction n'est assumée entièrement que par la signature électronique), elle insiste par contre sur le côté informatif. Cette observation est valable notamment pour la signature automatique qui tend à reprendre une partie des fonctions de l'en-tête du courrier traditionnel. Ce dernier rassemble les données qui permettent d'identifier et d'obtenir des informations sur le sujet réel des actions engagées par l'écrit en question, soit l'entreprise, dont l'auteur humain n'est que représentant. Par rapport à l'en-tête du normolettre, la signature donne des renseignements à un niveau "micro", relatifs non plus à l'entreprise entière (données que nous pourrions qualifier de «macro»), mais à un de ses constituants, le signataire.

Les éléments retenus et l'ordre dans lequel ils apparaissent peuvent varier. Dans la CEMO, la signature constitue généralement un marqueur personnel et un signe autocentrique (Marcoccia, 2000). Dans le courriel d'entreprise, les signatures automatiques peuvent également comporter une typographie particulière : «(l)a couleur permet d'afficher ses goûts graphiques, d'ajouter une touche de personnalité ou de mettre l'accent sur certains éléments d'information » indique Armand (2011: 65), tout en suggérant prudemment de vérifier les normes de mise dans l'entreprise.

Cannivet (2009: 250-251) liste les usages commerciaux potentiels de la signature : ainsi, on peut par exemple y inclure les prix décernés à la société, un slogan publicitaire ou un lien "cliquable ", acheminant vers le site de l'entreprise, une page promotionnelle des produits ou des services offerts etc. La signature devient alors un véritable outil marketing, adaptable selon le destinataire : " vous pourrez ainsi cibler et proposer des informations adéquates en fonction du compte de messagerie, du langage (français, anglais...) ou tout simplement du contexte du message (prospection, demande d'information...) » (ibidem). Les signatures « commerciales » sont usuellement mises en relief par une police spéciale lui réservée ou isolées du texte par un cadrage.

Finalement, la signature peut contribuer à la dimension relationnell,e ce qui est nouveau dans la communication écrite d'entreprise : " pour votre destinataire, la signature reste une marque élémentaire de courtoisie » ( $d$ ' Humières, 2005 : 336). Cette fonction revient à la signature tapuscrite. En opposition à la signature numérique et à la signature professionnelle complexe aux formes développées et stables, la signature composée par le scripteur peut prendre des formes variées, allant des initiales par le nom et/ou le prénom à la signature complète ou une signature collective, p.ex. le nom du service («L'équipe Gestion offre fidélité»: Gajewska, 2013 : 238). Dans les messages rédigés en contexte professionnel, on constate un usage particulier: une sorte de dédoublement de la signature personnelle et formelle qui consiste à introduire les initiales avant la signature professionnelle pour la rendre moins impersonnelle (Crystal, 2001: 103). Le remplacement de la signature professionnelle par la signature tapuscrite marque une étape dans le développement de la relation. La signature peut aussi être abandonnée au moment d'entrer par les scripteurs dans une dynamique interactionnelle.

L'outillage technique offre des possibilités inconnues au support traditionnel, mais aussi impose des limitations et des contraintes. Le cas examiné ici in extenso montre la manière 
dont la technologie reconfigure le genre et comment les propriétés qui caractérisent un genre donné évoluent sous l'influence d'un facteur nouveau. Dans la même lignée, nous pouvons citer entre autres les procédés qui rendent plus aisée la lecture sur l'écran, et plus particulièrement dans la "fenêtre » où s'affichent les messages. Afin de faciliter la saisie de l'information, on préconise d'adapter l'organisation logique de la missive électronique à l'espace dans laquelle elle se manifeste et de commencer par l'essentiel, soit par "l'information la plus importante au point de vue de votre correspondant" (Page, 2005 : 44). Le plan en « pyramide inversée » diffère de celui du courrier commercial traditionnel, rédigée usuellement selon le schéma "passé-présent-futur » (cf.1.2). Les logiciels courriers permettent d'enrichir l'aspect visuel de la page grâce à une large panoplie de papiers à lettres (arrière-plans), polices, couleurs et autres enjoliveurs graphiques. Toutefois, leur emploi ne doit pas être gratuit, mais contribuer à une meilleure saisie de l'information. Ainsi, le courriel professionnel a instauré des conventions typographiques propres qui ont pour but d'assurer une circulation correcte entre plusieurs messageries.

\subsection{La technologie en tant que facteur qui empêche la stabilisation du courriel professionnel en genres fortement ritualisés}

Les propriétés techniques du médium distribuent différemment les rôles assignés à des éléments analogiques $d u$ paratexte traditionnel et électronique et introduisent des nouvelles conventions organisationnelles et typographiques. Un changement de pratiques communicatives n'interdit pas en lui-même la stabilisation en genres fortement ritualisés à scripts très contraignants ("genres institués de mode (1)», Maingueneau 2004), qui, à l'instar des lettres commerciales anciennes, pourraient être classées dans des recueils de «modèles de courriels » ou « courriels types ». Toutefois, de par les propriétés du médium, le courrier électronique est un objet qui se prête à des modifications et des reprises, donc par excellence instable.

En premier lieu, la réponse automatique et la transmission du message à un autre destinataire entraînent un reprise des informations relatives au contexte d'énonciation initial. Afin de différencier les deux niveaux énonciatifs, Cusin-Berche (1999) reprend à Genette $(1982,1987)$ la notion du péritexte, qui désigne les éléments du paratexte inséparables du texte (titres, mise en page, sommaire, date d'édition, notes, illustrations etc). Cusin-Berche réserve l'appellation de péritexte aux rubriques prédéterminées par le logiciel qui apparaissent dans l'en-tête, en gardant le terme paratexte à ces mêmes éléments lorsqu'ils se présentent comme inséparables du texte. Du point de vue du contenu, le péritexte est similaire au paratexte épistolaire par le fait de fournir des informations sur le destinataire, la date et l'heure d'émission, l'objet et les destinataires en copie. Dans le cas d'un document transféré, le péritexte renvoie à l'acte de la transmission car il mentionne l'auteur de cet acte, la date et l'heure de son réalisation et son (ses) destinataire(s). Le paratexte qui figure dans le corps du texte correspond alors au péritexte de la situation d'énonciation première, en évoquant l'énonciateur premier, la date et l'heure de l'envoi au destinataire initial.

19 Le jeu entre le para- et le péritexte montre le courriel comme un objet complexe, dont les éléments changent de sens rien que par sa transmission à des destinataires autres que le (s) récepteur(s) initialement prévu(s). La communication numérique présente des spécificités textuelles propres. Les messages électroniques sont très souvent des 
plurilogues qui empruntent à une multiplicité de sources énonciatives. Par rapport au support papier immuable, le support électronique permet la manipulation des textes obtenus. L'usage de la procédure de transfert, la fragmentation des textes initiaux, leur insertion dans des textes ultérieurs sous forme modifiée ou accompagnés de commentaires métadiscursifs sont à l'origine des difficultés à identifier les sources énonciatives, mais aussi d'une absence de lisibilité.

"Dans une conversation en face à face, vous avez au moins une chance de corriger le tir et d'aider votre interlocuteur à vous comprendre. Si vous devenez confus à l'écrit, le destinataire ne fera pas longtemps l'effort de vous suivre dans vos élucubrations » (Brounstein \& Balique, 2011 : 251). Ainsi, la cohérence devient un élément crucial du texte. Les idées développées dans une lettre "pro» doivent être classées dans un enchaînement logique, suivant une progression adaptée à l'objectif. Le plus souvent on indique d'organiser la présentation des faits dans un ordre chronologique: passé présent - futur, ce qui qui correspond à l'introduction, au développement et à la conclusion du texte. Considérant ce schéma applicable à quasiment toutes les lettres, Fayet $(2006: 3)$ y voit le guide méthodologique de toute rédaction.

Le courrier part du principe de remplacer, dans la mesure du possible, le dialogue des textes par la reprise de l'information initiale et son inclusion dans le texte de la réponse. On est donc moins dans la logique du dialogue, où les procédés de cohésion lient les interventions dans le cadre d'un échange, mais dans un dialogue de textes écrits qui, tout en entrant en interaction avec d'autres textes, essaient d'être autosuffisants et former des unités sémantiquement complètes. Cette interdiscursivité n'est pas masquée, mais mise en relief par des références explicites afin de faciliter l'interprétation («En réponse à votre courrier du... »). Les préceptes rhétoriques et stylistiques du courrier traditionnel, stabilisés en des plans rhétoriques et des expressions pré-formées, étaient orientés sur le lecteur. Par la vitesse du transfert des messages, un échange de courriels donne l'illusion d'entrer dans une perspective dialogique. Comparé à la lettre traditionnelle qui devait être conçue comme un texte autosuffisant et complet, un courriel en appelle plus facilement le recours à un autre par l'anaphore, les procédés de thématisation ou les reformulations. Un maniement maladroit de ces procédés risque de rendre le texte opaque : sans l'aide de la structure rhétorique traditionnelle, un scripteur malhabile peut provoquer une surcharge cognitive de la part du lecteur.

Les procédés de reprendre les textes antérieurs dans leur totalité ou de les manipuler les tronquer ou y insérer des commentaires (courriers "palimpsestes») - sont mis au service de l'économie discursive. Cependant, dans le cadre de la présente réflexion, ils nous intéressent avant tout par le fait de gêner la stabilisation de l'expression libre en celle ritualisée, soit l'émergence d'un genre. En effet, les scripteurs entrent ici dans une dynamique qui est souvent rapprochée par certains égards aux échanges oraux. Ceci nous incite à nous pencher sur les origines du genre, c'est-à-dire sur les conditions qui imposent à un genre donné ses traits particuliers.

\section{Des changements de pratiques aux changements génériques}

23 Les écrits professionnels sont rédigés dans un contexte actionnel fort marqué par des normes sociales et souvent répétitif, ce qui appelle naturellement le concept du genre. 
Dans la Genre Analysis de tradition anglo-saxonne comme dans la perspective bakhtinienne d'expression française, le genre apparait comme une action sociale typique. Il semble logique que les changements des pratiques entraînent des changements génériques : regardons donc de près l'impact des nouveaux moyens de transmission de l'information sur la communication écrite en entreprise.

\subsection{Le genre comme une action sociale typique}

la suite se stabiliser en un répertoire de catégories normatives, soit de genres. Pour Moirand (2003 : 20), le genre est

une représentation socio-cognitive intériorisée que l'on a de la composition du déroulement d'une classe d'unités discursives, auxquelles on a été exposé dans la vie quotidienne, la vie professionnelle et les différents mondes que l'on a traversés, une sorte de patron permettant à chacun de construire, de planifier et d'interpréter les activités verbales ou non verbales à l'intérieur d'une situation de communication, d'un lieu, d'une communauté langagière, d'un monde social, d'une société (Moirand, $2003: 20$ ).

"Comme toute parole socialisée, le langage au travail n'échappe pas au formatage institutionnel en genres discursifs" (Lacoste, 2001: 33). Les genres routiniers professionnels cristallisent autour d'une besogne : dans une situation répétitive, l'employé doit réagir de manière à obtenir le résultat souhaité. Etant donné qu'il crée des énoncés identiques dans des situations identiques, le locuteur peut réutiliser les stratégies et les ressources qui, dans les occurrences antérieures, lui ont rendu service. L'organisation des genres professionnels reflète la dimension référentielle et contextuelle de l'action du sujet parlant. Bhatia et Bhatia (2007 : 264) voient dans les genres « the most common forms of realisations of professional discursive activities ». Ainsi, les genres apparaissent comme une composante essentielle des pratiques du métier.

Une séquence d'activités correspondant au traitement d'un « cas professionnel », soit aux activités (verbales et non verbales) à entreprendre, pourrait être associée aux « schémas » ou « scripts ». Toutefois, Bhatia (1993 : 21) soulève que la théorie de Schank et Abelson se réfère à l'accumulation d'un savoir individuel, alors que la perception du genre en tant que structure cognitive reflète un savoir partagé, propre à une communauté discursive donnée. Bargiela-Chiappini et Nickerson (1999: 3) soulignent « the inherently social nature of writing, it relatedness to other social activities and its power to shape the way human life unfolds through purposeful, personal acts of intervention ». Le genre devient alors le point de rencontre entre l'individuel et le social, les objectifs personnels et l'action stéréotypée : « the operational site of joint, reproductible social action, the nexus between private and public, singular and recurrent, micro et macro » (Miller, 1994 : 74).

Les contraintes sociales qui pèsent sur l'activité verbale dans une situation sociohistorico-culturelle déterminée se stabilisent en des paramètres qui régissent le(s) genre (s) respectif(s). Vu que les situations occasionnant la rédaction du courrier d'entreprise étaient typiques et répétitives, la codification des comportements langagiers efficaces a donné en résultat ce que Maingueneau (2004) appelle « genres institués de mode (1) », pas ou peu sujets à variation. Dans les manuels de «courrier commercial » ou "courrier d'entreprise », les structures rhétoriques des genres particuliers sont usuellement présentées en tant que «lettres-type » («modèles de lettres»), soit décomposées et examinées. Dans ce deuxième cas, un plan exprimé en termes de fonctions est suivi de 
conseils d'ordre divers (notamment sur les stratégies qui facilitent la réalisation des objectifs visés par ce genre de lettre) et d'expressions « routinisées » utiles lors de la mise en texte.

Une telle manière de procéder correspond à la méthodologie proposée par Swales (1990, 2004) et Bhatia (1993) dans le cadre de la Genre Analysis, où la structure rhétorique du genre apparaît comme un modèle cognitif pour l'interprétation et la production des textes génériques propres à une situation donnée. Des entités prototypiques (moves), ayant des fonctions rhétoriques particulières, contribuent à la réalisation du but visé par le genre en question. Les schèmes compositionnels d'un genre donné accusent des différences mineures, ce qui peut être examiné sur l'exemple des plans des lettres de réclamation relatives à la livraison.

Figure 1. Quelques modèles de lettres de réclamation

Début de la lettre : accuser réception de la livraison

Développement : détailler la réclamation

Formule de conclusion : demander réparation

Formule de politesse

(Nishimata, 2002: 68)

AR de la livraison; remerciements

Constatation du problème (quand ? quoi ? combien ?)

Demande de réparation (réparation intégrale ou proposition)

Attente de la décision du fournisseur

(Girault, 1996 : 78)

1. Accuser réception de la livraison en précisant la date

2. Signaler l'erreur ou confirmer les réserves émises lors de la livraison (...)

3. Demander réparation du préjudice et informer du renvoi des marchandises ou demander instructions au fournisseur (...)

4. Émettre le vœu que le problème ne se reproduira plus

(Bas \& Hesnard, 2005 : 36)

Les divergences observées correspondent à des différences des praxéogrammes, soit des actions à entreprendre au moment de la constatation de l'erreur : laisser la décision quant aux modes de réparation entièrement dans la gestion du fournisseur (Nishimata, 2002 : 68), proposer des solutions alternatives (Girault, 1966: 78) ou bien renvoyer la marchandise sans consultation (une des possibilités envisagées dans le plan de Bas et Hesnard, 2002: 36). Ainsi, le texte de la réclamation génère non seulement un texte suivant, mais aussi les actions qui vont s'ensuivre.

\subsection{Communiquer par écrit au travail : des pratiques reconfigurées}

Les actions professionnelles, tout comme les genres qui en résultent, ne constituent pas des événements uniques (tels des maillons isolés), mais s'inscrivent dans des séquences (" syntagmes ») d'actions. La connaissance de ces «maillages de pratiques » constitue une partie essentielle de la compétence professionnelle. Il en était conscient, l'auteur d'un des premiers manuels pour la rédaction du courrier commercial : «I have been careful to select 
simply transactions, and have in many cases, given the entire correspondence relation to them, in order to show distinctly the common routine of business " (Anderson, 1873'3).

L'ensemble des genres disponibles pour une communauté professionnelle donnée reflète non seulement les relations entre les acteurs, mais aussi la logique de procéder et de communiquer dans un secteur d'activité. Ainsi, selon Devitt (1991: 340) il est possible de dire que le système ou le répertoire des genres (genre set) accomplit le travail dans les situations de communications récurrentes, tout en contribuant à la stabilisation de cellesci. On peut donc le rapprocher du praxéogramme modélisant des représentations cognitives expériencielles, soit au "schéma d'actions verbales ou non verbales qui correspond à la représentation cognitive intériorisée que l'on a du déroulement d'une interaction" (Moirand dans: Charaudeau \& Maingueneau, 2002). Ce concept est de grande utilité pour l'analyse des textes plus ou moins normés, donc de genres discursifs divers : en effet, si une situation en mène à une autre, de même le texte routinisé qui y est créé en appelle un autre. Reconstruire une maillage de pratiques, dont pratiques discursives, équivaut à reconstruire la logique professionnelle d'un métier ou d'un poste. En entreprise, les procédures à suivre explicitent les opérations à entreprendre en vue d'atteindre un résultat ou un objectif souhaité, conceptualisant ainsi le praxéogramme respectif. Ainsi, les modèles d'action langagière écrite témoignent des manières de percevoir le courrier en tant qu'activités imbriquées dans la pratique quotidienne du métier.

Le noyau dur du courrier commercial sont les missives échangées à l'occasion de l'achat/ vente d'un produit ou d'un bien. Bhatia (2005: 39) organise les lettres professionnelles relatives à la commande en chaîne, allant des lettres de vente au règlement.

Figure 2. Chaîne des lettres d'affaires (Bhatia 2005 : 39)

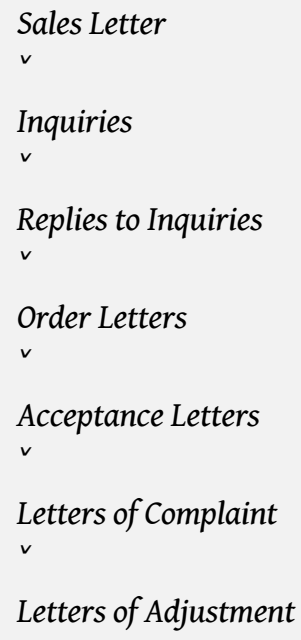

Cette représentation sous-entend que la totalité des interactions verbales se limite à des échanges écrits. Bhatia est ici dans une vision du traitement de la commande d'avant l'invention du téléphone, donc au moment où l'on rédigait les premiers manuels de rédaction professionnelle, tel le Practical Mercantile Correspondence d'Andreson cité précédemment. 


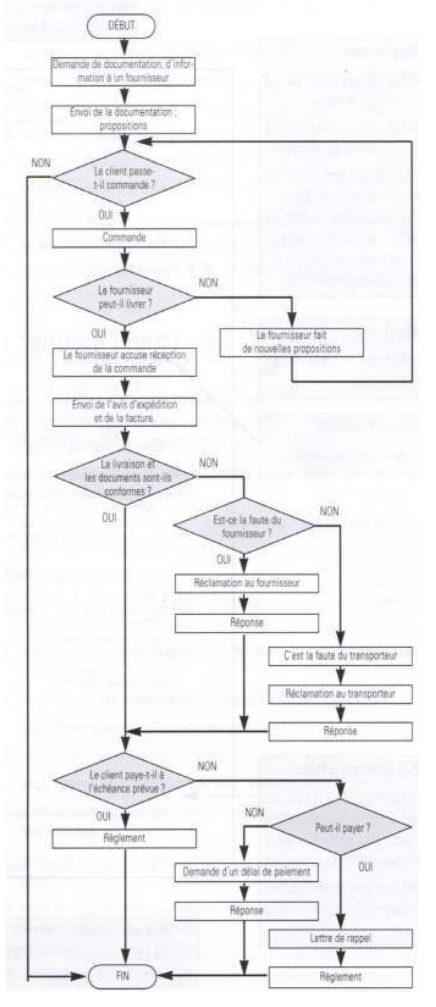

L'étude des organisations a élaboré des manières plus complexes qui permettent de figurer les procédures, à savoir les logigrammes. Ce type particulier d'organigramme, visualisant l'enchaînement des actions grâce à un code sémiologique propre, a été adopté également par certains auteurs de manuels de communication professionnelle, entre autres Danilo et Pelfornis (1993 : 53). À la différence du modèle unidirectionnel de Bhatia, leur visualisation du courrier orienté autour de la commande prévoit des bifurcations en fonction des données contextuelles. Toutefois, là encore, les (inter)actions écrites se déroulent une après l'autre dans une suite (chrono)logique, même si dans un circuit présenté de manière ramifié. De même, le schéma prend en considération uniquement des échanges écrits.

Les deux représentations témoignent du temps où non seulement les lettres commerciales, mais aussi les circonstances dans lesquelles elles étaient échangées étaient routinisées et prévisibles. Conformément à l'adage scripta manent, verba volant, les arrangements concertés par téléphone devaient être confirmés par une lettre du canon usuel, dans laquelle les échanges oraux étaient remplacés par une des routines rituelles («En confirmation de notre entretien téléphonique du ...,»). Par conséquence, le jeu complexe des négociations était réduit dans sa version écrite aux conclusions, dont la forme ne témoignait en rien des interactions verbales en amont. Ainsi, l'imprévu et la " parole fraîche » n'appartenaient qu'aux échanges oraux, tandis que l'écrit était censé constituer la preuve juridique des résolutions finales. Dans un contexte pareil, le courrier pouvait se restreindre à quelques genres routinisés et assurant un nombre de fonctions limitées.

Dans sa fonction d'outil bureautique, le courriel a remplacé non seulement le courrier papier, mais aussi, partiellement, le téléphone. Si le temps de saisie limite la vitesse du 
pseudo-dialogue électronique par rapport à celui oral, le courriel possède l'avantage incontestable d'être moins intrusif et dérangeant. D'autre part, la possibilité d'apporter des notes en palimpseste constitue une facilité importante, par laquelle il dépasse aussi bien le téléphone que le courrier.

Un corpus de courriels produits en contexte professionnel (Gajewska, 2013) atteste plusieurs échanges qui se font en quelque sorte "en périphérie» du schéma offre/ commande tel qu'il a été présenté supra. Un ensemble de 12 courriels constitue un exemple particulièrement intéressant. Quatre participants (un Polonais, deux Français et un Suisse), cadres supérieurs et ingénieurs y consultent les détails de l'offre pour le forage d'un tunnel dans les Alpes. Les courriels étaient échangés en tandems. Le premier engageait l'ingénieur polonais et son homologue français, le deuxième impliquait également le Polonais, cette fois-ci avec le Suisse chargé du côté financier de la commande. Les remarques étaient annotées en rouge dans la version provisoire de l'offre, soit sur des fragments de l'offre rapportés dans le texte des messages. Tous les courriels étaient transmis en copie au supérieur de l'entreprise franco-suisse. Grâce à l'option de répondre à tous les partenaires, celui-ci a contesté le prix d'une procédure technique, demandant de lui trouver une solution alternative moins onéreuse. Cette remarque a entrainé l'intervention de l'ingénieur français qui a signalé la nécessité de consulter un expert technique. Une heure et demie plus tard, son courriel relate non seulement les conclusions de l'entretien qu'il a eu avec un collègue travaillant dans le même établissement, mais aussi avec l'ingénieur polonais, avec qui il a traité par téléphone. Pendant le temps où le cadre technique français était pris ailleurs, le Polonais a apporté des commentaires sur la partie non contestée de l'offre. Cependant, une clause a suscité ses doutes, de sorte qu'il a consulté son PDG (ce dont il témoigne dans le courriel), lequel PDG a demandé d'éclaircir le point relatif à l'hébergement des ouvriers polonais. Les modalités de cet hébergement ont été traités avec le Suisse, en parallèle avec les consultations techniques (interrompues par les consultations orales) reprises. Le dernier courriel s'achève sur l'engagement de l'ingénieur français d'apporter toutes les modifications nécessaires sur la version définitive de l'offre et de l'envoyer le lendemain à toutes les personnes concernées. Et cela a été fait.

Le tissu interactionnel présenté supra se prête difficilement à une conceptualisation graphique par logigramme, même dans les cas où nous n'envisagerions que les actions d'un seul des protagonistes impliqués. L'interaction, mixte, est composée de séquences écrites et orales, effectuées simultanément ou imbriquées les unes dans les autres. Une partie des échanges examinés se chevauchent : cependant, à la différence de l'interaction orale, l'échange simultané des messages électroniques et/ou entretiens oraux ne gêne pas la transmission des messages qui occupent des canaux distincts. Richer (2008) évoque le rôle toujours en augmentation de la coopération et de la coordination qui, comme le démontre l'exemple supra, se font désormais en grande partie par courriel. Ainsi, les activités de communication se voient reconfigurées. Examinons les effets qui en résultent pour l'abandon et la naissance des genres épistolaires nouveaux.

\section{Le courriel : un champ générique en gestation}

Le changement, ou même éclatement des praxéogrammes, dû à des manières nouvelles de communiquer, laisse présager que nous aurons affaire à des formes de communication nouvelles. Le répertoire des types reste stable jusqu'au moment où la communauté 
n'identifie une situation pour nouvelle et exigeant par conséquence une réponse rhétorique innovante. Souvent, cette « réponse " évolue à partir de types existants. En est-il de même pour le courrier et le courriel?

\subsection{Le courrier traditionnel : un système de genres à ton et style prédéfinis} système stable qui permettait de gérer les contacts usuels avec les partenaires extérieurs de l'entreprise et la transmission des informations au sein de l'établissement. La stabilité (pour ne pas dire : fossilisation) des manières de communiquer se reflète entre autre dans la manière de conceptualiser cette communication. En effet, les divers recueils utilisaient les mêmes principes pour organiser les genres épistolaires. Les classements usuels en courrier externe/interne, selon le sens de transmission ou selon les moments de la vie de l'entreprise introduisaient des "champs génériques» (terme de Rastier, 2001, adapté à l'analyse des discours professionnels par Poudat, 2006), qui permettaient de contraster les genres appartenant à un même champ pratique ou lieu institutionnel.

La théorie pragmatique a démontré que chaque énoncé est étroitement lié à un ensemble de stratégies communicatives. Par conséquence, la fonction pragmatique (associée à l'intention de communication) est considérée comme un des critères principaux pour la distinction d'un genre, ce que démontrent entre autres Askehave et Swales (2000). Suite à l'étude d'un ensemble de lettres sous forme papier, Fayet (2006) dégage cinq actions dominantes qui mettent le rédacteur dans des positions différentes vis-à-vis du destinataire, schématisées par l'acronyme PEJRO: présenter, enregistrer, justifier, réclamer et ordonner. Pourtant, la classification fonctionnelle apparaît rarement comme critère unique. Certes, par rapport aux autres critères d'organisation des genres du courrier, la fonction pragmatique se pose en clé de voûte. Toutefois, les variantes référentielles (réclamation pour retard de livraison, avaries ou manquants) ou situationnelles (AR de la commande ou AR avec problème, les rappels successifs de règlement) la déclinent en sous-genres en bas. En haut, les divers genres de courrier professionnel organisent le champ générique dans lequel ils coexistent en constellations plus ou moins complexes, déterminées selon la zone de diffusion, les moments de la vie de l'entreprise ou les étapes de la procédure vente/commande.

Aux catégories génériques se combinent parfois les notions de ton, de registre ou de style. Ces appellations, couramment utilisées, entretiennent des relations variant d'une source à l'autre. Le ton désigne usuellement les émotions ressenties par le scripteur par rapport au destinataire manifestes au niveau du texte. Dans ce sens, il est utilisé entre autres par Fayet (2006: 17-27) pour classer les formules proposées et va du «très aimable » par « aimable/neutre » et «irrité » au "sec ». Dans un autre manuel de la même auteure (Fayet \& Nishimata, $2009:$ 50-51), dans le sous-chapitre «registre utilisé par la langue professionnelle", les "tons utilisés dans les écrits professionnels" se déclinent en " colère ", " exposition de faits " et " amabilité ». Fayet et Nishimata listent par la suite des expressions correspondant à chacune de ces catégories. Néanmoins, les auteures constatent qu'à l'écrit la gamme des possibilités est assez réduite et l'éventail des nuances étroit.

Le moment d'intensité de ton extrême est l'expression de la colère; le moment le plus faible, celui de l'amabilité ; au centre, des mots qui transmettent simplement

Pratiques, $171-172$ | 2016 
des faits. (...) Cependant, les verbes qui permettent de passer de la colère à l'amabilité n'offrent entre eux que des faibles nuances à l'écrit : le ton général est d'une extrême modération apparente. (ibidem : 50-51).

Tout en étant une catégorie transversale par rapport au courrier privé, ce critère ne semble pas se combiner librement aux sous-genres du courriel professionnel, vu que la fonction principale de la lettre impose le ton à utiliser. Dans le modèle de Fayet (2006), l'ossature du texte est fournie par l'objet (les cinq actions dominantes: présenter, enregistrer, justifier, réclamer ou ordonner) et le ton (du très aimable au sec) qui en dérive. Néanmoins, ce dernier ne s'accorde pas avec n'importe quelle fonction pragmatique, mais en découle par voie de conséquence : "on n'utilise pas le même ton pour se justifier ou pour donner un ordre " (Fayet, 2006: 7). Dans les grilles récapitulatives qui présentent les données de base relatives aux (sous-)genres de lettres d'entreprise proposés, le ton est suggéré d'emblée et ne se prête pas à des modifications.

Figure 4. Grille récapitulative des écrits autour de « la gestion du personnel » (Fayet 2006 : 123)

\begin{tabular}{|l|l|l|l|}
\hline $\begin{array}{l}\text { Objet de la lettre } \\
\text { ou message électronique }\end{array}$ & Rédacteur & $\begin{array}{l}\text { Action } \\
\text { dominante }\end{array}$ & Ton \\
\hline 31 Demande de service à un cabinet de recrutement & Client & Présenter & Aimable \\
\hline 32 Convocation à un entretien d'embauche & Employeur & Justifier & Aimable \\
\hline $\begin{array}{l}33 \text { Réponse négative à une candidature spontanée } \\
\text { (conservation de CV) }\end{array}$ & Employeur & Justifier & $\begin{array}{l}\text { Très } \\
\text { aimable }\end{array}$ \\
\hline $34-$ Réponse négative après un entretien d'embauche & Employeur & Justifier & Aimable \\
\hline $\begin{array}{l}35 \text { Réponse positive adressée à un salarié } \\
\text { démissionnaire souhaitant ne pas effectuer son préavis }\end{array}$ & Employeur & Enregistrer & Neutre \\
\hline $\begin{array}{l}36 \text { Convocation à un entretien en vue d'un } \\
\text { licenciement }\end{array}$ & Employeur & Ordonner & Sec \\
\hline $37-$ Lettre de licenciement & Employeur & Justifier & Irrité \\
\hline 38 Certificat de travail & Employeur & Présenter & Neutre \\
\hline 39 Formulaire de reçu pour solde de tout compte & Employé & Enregistrer & Sec \\
\hline
\end{tabular}

Une autre catégorie potentiellement transversale au champ générique du $C R$ professionnel semble le style. Cette notion apparait en contexte d'une écriture claire et soignée. Pour Bas et Hesnard (2002 : 18), un style commercial approprié égale clarté, concision, courtoisie et soin du langage. Les manuels prônent un style " simple, direct " (Girault, 1996:12), ce qui correspond essentiellement à une seule idée par paragraphe ou même par phrase et des phrases si possible courtes (16 mots en moyenne pour Girault, $1996: 12 ; 20$ pour Bas et Hesnard, 2002:18; 10 à 24 pour Nishimata, 2006:29). Cette conception du style est bien loin du «style individuel». En effet, si l'apprentissage scolaire de la langue favorise la réflexion, prédispose aux tournures complexes et essaie « de nous faire rédiger de la littérature, de démontrer notre maitrise de cette langue si 
difficile » (Lelli, 2003: 35), pour les écrits professionnels l'objectif fondamental est de favoriser l'action appropriée. La recherche de l'originalité pourrait nuire à l'efficacité recherchée en tout premier lieu.

Ainsi, les catégories transversales aux genres du courrier professionnel traditionnel avaient peu d'importance en pratique didactique. Le style et le ton pouvaient apparaître dans les préfaces aux recueils des modèles de lettres, néanmoins le courrier "papier " était abordé par l'entrée du genre. Vu que les situations d'écrit étaient récurrentes et prévisibles, les genres du courrier traditionnel étaient analysés et enseignés essentiellement à partir de la situation professionnelle qui les engendraient et à laquelle ils permettaient de faire face. Quant au style et au ton, ils étaient éventuellement envisagés comme des caractéristiques d'un genre donné, que pourtant on n'aura pas à modifier, car ils sont en quelque sorte inhérents à ce genre.

\subsection{Le " courriel commercial »: un ensemble de genres décliné en registres}

En comparaison avec les champs du courrier d'entreprise et du courrier commercial, les tentatives de classer les genres des méls sont relativement peu nombreuses et basées sur des catégories diverses, ce qui corrobore l'idée que le courriel est une forme d'expression in statu nascendi.

Certains classements ont été forgés sur des modèles anciens. Nishimata (2011: 2-4) organise ses « 100 e-mails professionnels » selon les champs génériques appliquées dans ses ouvrages consacrés au courrier classique, c'est-à-dire les relations fournisseur-client, relations avec les banques, les assurances, le personnel, l'administration ou les relations internationales. Cependant, cette typologie semble faire couler une substance nouvelle dans des fontes ancielles: dans une optique pareille, le courriel ne serait qu'un courrier qui voyage plus vite, avec peut-être des légères modifications de mise en page dues aux exigences du médium. Une pareille approche est visible notamment dans les ouvrages qui juxtaposent le courrier traditionnel et électronique, dont les Modèles types de lettres et courriers électroniques de Fayet (2006). Les fonctions du courriel, schématisée par l'acronyme PAIX, y sont différenciées de celles du courrier. Si le CR est fait pour présenter, enregistrer, justifier, réclamer et ordonner (PEJRO), le courriel est axé sur la résolution d'un problème $(\mathrm{P})$, demande la réalisation d'une action $(\mathrm{A})$ ou transmet une information (I), sans compter les formes mixtes (X). Toutefois, les exemples de courriels n'accusent que des différences mineures par rapport aux courriers respectifs. Cette attitude de modéliser l'expression électronique sur cette traditionnelle est particulièrement marquée dans les guides de rédaction élaborés par des auteurs ayant l'habitude des médias anciens.

48 La liste d'Adam et al. (2008: 29-32) témoigne des phénomènes saisis sur le vif et mérite de ce fait d'être citée in extenso, car les dénominations apportées au courriel résultent des catégorisations opérées par les utilisateurs des genres en question. Elle juxtapose des catégories disparates: le courriel ${ }^{4}$ informatif (dont le but est de transmettre une information, sans forcément demander une action immédiate au retour), le courriel question, le courriel opérationnel (p. ex. une procédure d'inscription ou d'achat, souvent automatisée), le courriel privé, le courriel blague, le courriel remerciement (proche du courriel opérationnel, envoyé en confirmation à une inscription ou un achat), le courriel " reply», le courriel en copie (envoyé " pour information » à des personnes intéressées 
qui n'en sont pas pourtant le destinataire principal), «le mail parapluie» (courriel à contenu délicat qui « met en copie toute votre hiérarchie afin de vous protéger en cas de retour de bâton »), « le mail je règle mes comptes » (« envoyé sur le coup de la colère : on écrit ce qu'on n'a pas pu dire... »), le courriel réponse automatique (" out of office »), le courriel mailing, le courriel newsletter, le spam, l'hoax (courriel qui diffuse une information fausse ou périmée et qui incite le destinataire à diffuser la fausse nouvelle à son tour), le courriel lettre (où toute l'information est contenue dans le texte de la lettre électronique), le courriel enveloppe (où le mèl n'est qu'un canal pour transporter l'information qui se trouve dans un fichier joint en annexe).

49 Les genres qui peuvent être rapprochés aux genres traditionnels (comme le «mail enveloppe » aux lettres accompagnant les factures ou autres documents) à part, une grande partie de textes (nous hésitons ici à parler de genres) sont distingués en fonction de critères extratextuels. N'importe quel courriel peut être transmis « en copie », et la distinction entre les courriels envoyés « pour information » et pour protéger sa peau « au cas où » (« le mail parapluie ») ne dépend que des intentions du destinataire, ou plutôt, selon la distinction de Cusin-Berche (1999), du transmetteur ou médiataire. La réponse automatique (qui informe que l'employé est temporairement absent, p.ex. en mission de service) est un genre écrit nouveau, auquel on pourrait attribuer cependant un antécédent vocal : le répondeur automatique. Dans la même lignée nous pourrions classer la blague, elle aussi renvoyant à l'oral. Un autre genre récent est le courriel dont tout le contenu se situe dans la ligne objet, ce qui n'est pas sans conséquence au niveau de sa forme langagière. Le courriel «je règle mes comptes» attire notre attention sur le fait que le courrier papier ne prévoyait pas une forme pareille, ceci non seulement du fait des propriétés du canal (le temps de rédiger et d'aller poster la lettre permettant d'habitude de retrouver des idées claires), mais aussi en vertu du système qui n'admettait pas des réponses directes et malpolies. Les maillages de pratiques stabilisées qui traduisaient la logique des actions professionnelles excluaient des cas pareils.

50 L'exemple examiné supra montre que les typologies ordinaires ne sont pas toujours les mieux placés pour déterminer tel ou tel autre genre. Néanmoins, cette liste met en évidence que le courriel électronique va selon toute vraisemblance se scinder en genres d'une manière pour le moins partiellement différente des partages opérés dans le champ du courrier papier. Une quantité considérable de genres potentiels doit leur émergence non seulement aux potentialités nouvelles du canal de communication, mais également à l'application de l'écrit aux contextes réservés autrefois aux rencontres de visu (missives échangées avec les collègues) ou par téléphone. Bhatia (1997: 191) définit les «genres enchâssés" (genre embedding) comme des cas dans lesquels une forme générique particulière est utilisée comme modèle pour donner expression à une autre forme générique conventionnelle distincte. Dans un monde en évolution, les genres professionnelles s'ouvrent à de nouveaux buts communicatifs, ce qui entraîne un « mélange de genres » (genre mixing : Bhatia, 1997).

51 Face aux problèmes que posent des genres pas ou peu stabilisés, ou qui adoptent des formes mixtes, des solutions didactiques pertinentes pourraient être cherchées du côté des catégories plus larges : faute de pouvoir enseigner les règles qui régissent les genres, on miserait alors sur le style et le ton. L'analyse du style administratif (dans une acception plus proche du type de discours que du style personnel) occupe effectivement dans les guides de rédaction du courriel professionnel une place plus importante que dans les recueils de lettres-types. Quant au(x) ton(s) des missives, les dimensions du 
présent article ne nous permettent pas de développer l'analyse des conséquences qu'a eu l'ouverture du courrier (notamment interne) à des situations à forte dominante émotionnelle. Par contre, examinons une nouvelle donne qui apparaît par rapport à l'écrit professionnel, à savoir la variation de registres.

Le premier chapitre de la Rédaction des courriels efficaces d'Armand (2011) porte un titre significatif : «choisir son style en fonction du destinataire ». L'auteure précise ce qui se cache derrière cette notion :

«D'un destinataire à l'autre, certains éléments peuvent différer. Par exemple: le ton, la présence ou l'absence d'humour, la ponctuation, les explications plus ou moins détaillées, la signature, la présence ou l'absence d'une mise en contexte » (Armand, 2011:8).

Armand (ibidem: 4-8) fournit six exemples de lettres illustrant ces différences en pratique: six variantes sur le thème de demande de fichiers afin de mener à bien le travail sur un dossier important.

Figure 5. «Choisir son style en fonction du destinataire » (Armand, $2011: 4-8$ )

\section{Haute direction :}

Monsieur le vice-président, Je travaille présentement sur le dossier des commandites.

Afin de mener à bien le projet, nous aurions besoin du fichier Excel sur lequel vous avez travaillé la mois dernier et ayant le nom « Prévision de ventes, avril-mai 2011 ». Cependant, si nous voulons réussir à livrer à temps, il nous faudrait recevoir ce fichier avant la fin de la journée.

Bien que le délai soit très serré, nous comptons énormément sur votre appui pour faire de ce projet une réussite.

En espérant avoir le plaisir de vous compter parmi nos collaborateurs stratégiques, veuillez accepter à l'avance nos plus sincères remerciements.

Luc Tremblay

Chargé de projet senior

\section{Collègue:}

Salut Roger!

Je travaille en ce moment comme un fou sur le dossier des commandites.

Pourrais-tu m'envoyer le fichier Excel sur les liquidités des mois d'avril et mai 2011?

Si tu pouvais me le faire parvenir d'ici la fin de la journée, ça serait formidable. Je dois terminer l'analyse et remettre le document lundi matin !!! Merci d'avance,

Luc

Ami :

Salut Roger!

Je sais, il est tard...

Si tu attrapes ce message avant demain matin, pourrais-tu m'envoyer ton fichier Excel sur les liquidités des mois d'avril et mai 2011 ?

Je dois terminer une importante analyse et remettre le document lundi matin !!! 
Je ne peux pas t'en dire plus car le projet est confidentiel, mais si tu pouvais m'envoyer le fichier, ça m'éviterait de m'arracher les cheveux (j'en ai déjà pas beaucoup !)

Merci mon vieux,

Luc-le-chauve

Un genre nouveau - la demande - s'inscrit ici dans un réseau de possibilités complexes par le fait de pouvoir être adressée à des destinataires à propriétés socioculturelles variées ( « haute direction », « employé », « client », « collègue », « fournisseur » et « ami), avec lequel le scripteur entretient des relations diversifiées quant aux rapports de pouvoir, liens institutionnels ou émotionnels. Le destinataire visé par un genre est également un facteur qui peut entraîner l'émergence d'un genre nouveau (Gillaerts \& Gotti, 2005: 11), ce qui est visible notamment en communication interne. Les praxéogrammes anciens prévoyaient la note de service (sens descendant) ou la communication orale (sens ascendant ou horizontal). Concurrençant la communication orale de visu et par téléphone, le courriel a ouvert l'écrit à des récepteurs nouveaux.

La demande écrite se faisait autrefois par une note de service ou une lettre externe, les deux ayant des propriétés formelles bien définies. La demande par courriel embrasse les deux cas et couvre en plus les appels téléphoniques informels. Parmi les facteurs génériques potentiels nous avons ainsi non seulement des fonctions nouvelles, mais aussi des destinataires qui n'étaient pas attestés préalablement. Du coup, le registre (ton, style) n'est plus associé de manière fixe au texte rédigé, comme il en était dans le courrier classique, mais devient une catégorie transversale qui, en quelque sorte, complique davantage la tâche rédactionnelle. Dans la grille examinée préalablement (fig. 4), le ton est juste une particularité qui caractérise le texte proposé en «modèle » unique, et non une des variantes possibles que ce texte pourrait prendre en fonction du destinataire, ou, en d'autres termes, une touche qu'on devrait lui donner en adaptant le genre au récepteur. Ainsi, le type de discours (dans l'acception française d'unité supérieure au genre) ou le registre (si l'on choisit la tradition conceptuelle anglo-saxonne) varie dans le cadre d'un genre unique, en ouvrant potentiellement sur des sous-genres.

\section{Conclusion}

Le champ générique du courriel est un terrain en gestation, où poussent des genres nouveaux. Ceci est dû non seulement aux propriétés techniques des messageries électroniques, mais surtout au bouleversement des anciens schémas actionnels. L'écriture électroniquement médiée au travail est devenue une activité discontinue, contribuant à la formation des suites d'activités souples, ouvertes à la "parole fraîche ». Cette évolution ira sûrement grandissant $\mathrm{du}$ fait de la démultiplication des technologies de communication, dont les outils (ordinateurs personnels, téléphones mobiles, tablettes...) et les formats (courriels, textos, échanges sur des réseaux sociaux...) ne cessent de monter en nombre. La communication écrite avec les partenaires externes de l'entreprise, autrefois limité à la lettre, a aujourd'hui de bien nombreuses facettes.

$57 \mathrm{Si}$, dans un premier temps, le courriel commercial se forgeait sur les genres du courrier traditionnel, peu à peu il prenait des formes nouvelles. En analysant les relations entre les genres, Swales (2004: 12-25) recense des "constellations de genres » (constellations of genres) diverses. Outre les « chaînages de genres » (genre chains) : séquences logiques et 
chronologiques de textes, où un texte en mène à un autre, il liste les " réseaux de genres " (genre networks) : un système de genres écrits et oraux qui traduit les logiques et les manières de faire à l'intérieur d'un domaine. A l'époque précédant les médias électroniques, les missives étaient censées laisser des traces écrites d'une action professionnelle. Dans certains cas particuliers, tel le courrier orienté autour de la vente/ achat, les genres épistolaires formaient des vrais systèmes qui, d'après Devitt (1991 : 340) cité en 1.2, permettaient d'accomplir le travail dans les situations de communications récurrentes.

Actuellement, les contextes qui appellent la communication écrite sont de loin plus nombreux. Faute de routine et de sédimentation à force de répétition pendant des dizaines d'années (ce qui était le cas du courrier papier), les formes d'expression qui émergent sont assez libres, ce qui augmente davantage le rôle des choix personnels. Le courrier d'entreprise était perçu comme un objet construit et réfléchi. « Pour fournir au destinataire du message tous les éléments d'information et pour lui en faciliter la compréhension, l'émetteur doit apporter le plus grand soin à la recherche des idées et à leur organisation» (Girault 1996: 10). Évidemment, la manière (et le degré) dont ces postulats étaient mis en pratique dépendaient de l'habilité du scripteur, mais aussi d'un apprentissage à partir des exemples qu'on ait jugés efficaces.

Le courrier électronique est un mode de communication nouveau, qui n'exclut et n'impose aucune forme scripturale codée socialement. En l'absence de normes génériques strictes, les scripteurs semblent s'ouvrir à des aspects conceptualisées en termes de registre, ton ou style individuel. Dans le courrier d'entreprise, le registre (formel) était envisagé comme un trait inhérent à un genre donné. Par contre, pour le moins une partie des genres du courrier électronique (notamment les genres nouveaux, qui manquent de routines stabilisées) se déclinent par registres, allant du formel à l'amical. Ils peuvent aussi, apparemment, se décliner par ton, ce dont témoignent les nombreux avertissements contre les courriels écrits sous l'effet d'une montée de colère. L'adoption des registres non-formels et l'expression des sentiments sont une nouveauté dans le courrier d'entreprise, traditionnellement focalisé sur le référentiel et censurant l'émotif, le conatif et le poétique. Finalement, contrairement au courrier traditionnel à formules ritualisées consacrées par l'usage et dépourvues d'originalité, la parole fraîche laisse plus de place aux variantes stylistiques individuelles et peut varier d'auteur à auteur.

60 Ainsi, les réponses génériques vont apparemment cristalliser dans une quantité limitée de situations de communication récurrentes, voire dans des champs institutionnels particuliers. Les genres formeront donc plutôt des placements individuels et temporaires, ce que Swales (2004:13) taxe d'ensemble de genres ("genre set») qui coexistent dans un champ générique donné, sans toutefois former de vrais systèmes, tel le courrier commercial d'autrefois. Comparés aux recueils de «lettres types» ou de «modèles de lettres ", les manuels pour l'acquisition de l'art du courriel professionnel prennent la forme de réflexion sur le style ou de conseils rédactionnels généraux. Face à l'écran, au lieu d'un modèle de texte prêt-à-porter, qui nous permettrait de tirer l'épingle du jeu au moindre effort, une réflexion consciente sur la communication et ses objectifs s'impose. 


\section{BIBLIOGRAPHIE}

ASKeHAVE, I. \& SWALES, J. M. (2000). « Genre Identification and Communicative Purpose : A Problem and Possible Solution ». Applied Linguistics 22, p. 195-212.

BARGIELA-CHIAPPINI, F. \& NICKERSON, C. (1999). «Business Writing as Social Interaction ». In: Bargiela-Chiappini, F. \& Nickerson, C. (eds), Writing Business : Genres, Media and Discourses. London: Longman, p. 1-32.

BHATIA, V. K. (1993). Analysing Genre. Language Use in Professional Settings. London: Longman. - (1997). « Genre-mixing in Academic Introductions ». English for Specific Purposes 16 (3), p. 181-195.

- (2005). «Interdiscursivity in Business Letter ». In: Gillaerts, P. \& Gotti, M. (eds), Genre Variation in Business Letters. Bern: Peter Lang, p. 31-54.

BHATiA, V. K. \& BHAтіA, A. (2007). « Global Genres in Local Contexts », in : V. K. Bhatia \& M. Gotti (éds), Explorations in Specialized Genres. Bern: Peter Lang, p. 263-281.

Charaudeau, P. \& MAingueneau, D. (éds) (2002). Dictionnaire d'analyse du discours. Paris : Seuil. CRYSTAL, D. (2001). Language and the Internet. Cambridge: Cambridge University Press.

CUSIN-BERCHE, F. (1999). « Courriel et genres discursifs ». In: Anis, J. (éd.), Internet, communication et langue française. Paris : Hermès Science, p. 31-54.

DEVITT, A. J. (1991). « Intertextuality in Tax Acconting : Generic, Referential and Functional »,. In: Bazerman, C. \& Paradis, J. (eds), Textual Dynamics of the Professions : Historical and Contemporary Studies of Writing in Professional Communities. Madison: University of Wisconsin Press, p. 336-357.

FAYET, M., NISHIMATA, A. (2009). Savoir rédiger le courrier d'entreprise. Paris : Éditions d'Organisation. GAJEWSKA, E. (2013). Courriel vs courrier : la communication écrite en français de la communication professionnelle au temps des nouvelles technologies. Lublin : Werset.

GenetTe, G. (1982). Palimpsestes. Paris : Seuil.

- (1987). Seuils. Paris : Seuil

GILLAERTS, P. \& GOTTI, M. (éds) (2005). Genre Variation in Business Letters. Bern: Peter Lang.

GUÉRIN, S. (2008). «L Les paratextes : approches critiques ». Loxias 20, En ligne : http://

revel.unice.fr/loxias/index.html?id=2110.

LAcoste, M. (2001). « Peut-on travailler sans communiquer ». In: Borzeix, A. \& Fraenkel, B. (éds),

Langage et travail, Communication, cognition, action. Paris : CNRS Éditions, p. 21-54.

MAINGUENEAU, D. (2004). « Typologie des genres de discours institués ». En ligne : http://

dominique.maingueneau.pagesperso-orange.fr/intro_topic.html.

MARCoccia, M. (2000). « La représentation du non-verbal dans la communication écrite médiatisée par ordinateur ». Communication et organisation 18, p. 265-274.

MILLER, C. R. (1994). « Rhetorical Community : The Cultural Basis of Genre ». In: Freedman, A. \& Medway, P. (eds), Genre and the New Rhetoric. London: Taylor \& Francis, p. 67-78. 
MOIRAND, S. (2003). «Quelles catégories descriptives pour la mise au jour des genres du discours? ». In: Kerbrat-Orecchioni, C. \& Traverso, V. (éds), Les genres de l'oral. Journée organisée le 18 avril 2003, Université Lyon II. EN ligne : http://gric.univ-lyon2.fr/Equipe1/actes/ journees_genre.htm. MOURLHON-DALlies, F. \& Colin, J.-Y. (1999). « Des didascalies sur l'internet? ». In: Anis, J. (éd.), Internet, communication et langue française. Paris : Hermès Science, p. 13-30.

NiSHimatA, A. (2006). Les 150 lettres et e-mails du créateur. Gualino Éditeur.

POUDAT, C. (2006). «Étude contrastive de l'article scientifique de revue linguistique dans une perspective d'analyse des genres ». Texto! 11 (3-4). En ligne : http://www.revuetexto.net/1996-2007/Corpus/Corpus.html.

RASTIER, F. (2001). Arts et sciences du texte. Paris : Presses universitaires de France.

RICHER, J.-J. (2008). « Le FOS ou une didactique du langage et de l'action ». Synergies Chine 3, p. 117-126.

SCHANK, R. C. \& ABELSON, R. P. (1977). Scripts, plans, goals and understanding: an inquiry into human knowledge structures. Hillsdale: Erlbaum.

SWALES, J.M. (1990). Genre Analysis. English in Academic and Research Settings. Cambridge: Cambridge University Press.

SWALES, J. M. (2004). Research Genres, Exploration and Application. Cambridge: Cambridge University Press.

\section{Guides pour la rédaction du $\mathrm{CR}$ et du CL professionnel cités}

ADAM, A., AUBERT, M., COUSSEMENT, A., MEULEMAN, F., \& PAY, T. (2008). Écrire et manager sa communication Web. Paris : Edipro Business \& Economics.

ARMAND, N. (2011). Rédaction de courriels efficaces. Québec: Presses de l'Univerité de Québec.

BAS, L. \& HESNARD, C. (2005). La correspondance commerciale francaise. Paris : Nathan.

CANNIVET, I. (2009). Bien rédiger pour le Web. Paris : Eyrolles.

BROUNSTEIN, M. \& BALIQUE, F. (2011). Communiquer efficacement pour les nuls. Paris : First Édition.

DANILO, M. \& PELFORNIS, J.-L. (1993). Le français de la communication professionnelle. Paris : CLE International.

FAYET, M. (2006). Modèles types de lettres et courriers électroniques. Pour une image de qualité de votre entreprise. Paris : Éditions d'Organisation.

GIRAULT, O. (1996). Les outils de la communication écrite d'entreprise. Courriers. Messages internes.

Paris : Foucher.

HUMIÈRES, I. d' (2005). Communiquer par écrit. Des documents professionnels efficaces. Paris : Larousse.

LELLI, A. (2003). Les écrits professionnels: la méthode des 7C. Paris : Dunod.

NISHIMATA, A. (2002). L'essentiel des formules types du courrier d'entreprise (lettres et e-mails). Paris : Gualino Éditeur.

NISHIMATA, A. (2011). 100 e-mails professionnels pour bien communiquer. Paris : Gualino Éditeur. 
PAGE, M. (2005). Courriels, mode d'emploi. Du bon usage des courriers électroniques. Paris : Éditions

WEKA.

\section{NOTES}

1. Nous y faisons abstraction des ouvrages dont les auteurs ont conçu antérieurement des guides pour la rédaction du courrier classique : l'examen des textes proposés démontre que leur expérience des usages de la communication écrite sur support papier les incite à appliquer les precepts de celle-ci aussi à la communication médiée par ordinateur.

2. Ils se distinguent d'ailleurs du courrier professionnel usuel au niveau formel, abandonnant le plus souvent la disposition du normolettre.

3. Anderson W. (1873): Practical Mercantile Correspondence. Milano: Bernardoni (cité par Del Lungo-Camiciotti, $2005:$ :125)

4. Dans le texte ici examiné, Adam utilise l'appellation « mail».

\section{RÉSUMÉS}

Communiquer efficacement par écrit en contexte et pour des besoins professionnels est un savoir-faire qui s'apprend. Pour cette raison, par le passé, dans les entreprises, la rédaction du courrier était allouée au personnel ayant une formation spécialisée (secrétaires ou adjoints). Avec l'avènement des logiciels de messagerie, savoir rédiger devient indispensable à un nombre de plus en plus large de professionnels. Or, si les rédacteurs du courrier traditionnel étaient assistés dans leur tâche par maintes guides ou recueils de « modèles de lettres ", les outils censés faciliter la rédaction du courrier électronique sont de loin moins nombreux.

Les lettres d'affaires traditionnelles, stabilisées en "lettres-type », étaient abordés par le biais du genre. Toutefois, cet angle d'attaque semble moins approprié à l'étude et à l'enseignement du "courriel d'entreprise ». Si les études de ses genres (ou sous-genres) particuliers s'avèrent pertinentes, les panoramas d'ensemble accusent souvent des points faibles ou se montrent plus ou moins discordantes. Une des raisons possibles d'un tel état de choses est que la constitution de la communication électronique en genre(s) n'est pas encore achevée. Devant de tels doutes, nous allons remonter à l'origine même du genre, soit à la situation - récurrente et typique - qui le génère. L'examen des conditions qui ont contribué à la formation du courrier dans sa version papier et de son équivalent numérique pourrait permettre d'estimer les chances que le courriel se stabilise en un ou plusieurs genres fixes, tels les genres du courrier traditionnel. Nous allons également examiner quelques concepts potentiellement utiles pour établir les typologies du courrier et du courriel professionnel (notamment genre network vs genre set), ainsi que les catégories considérées comme transversales, appliquées à ces deux domaines (ton et style vs registre). 
INDEX

Mots-clés : français sur objectifs spécifiques, didactique de l'ecrit, théorie des genres, courrier professionnel, courrier electronique

\section{AUTEUR}

\section{ELZBIETA GAJEWSKA}

Université pédagogique de Cracovie, Pologne 